\title{
Prevention and treatment of dry socket
}

\section{Abstracted from}

Daly B, Sharif MO, Newton T, Jones K, Worthington HV.

Local interventions for the management of alveolar osteitis (dry socket). Cochrane Database Syst Rev. 2012; 12: CD006968.

Address for correspondence: Luisa Fernandez Mauleffinch, Review Group Co-ordinator, Cochrane Oral Health Group, MANDEC, School of Dentistry, University of Manchester,

Higher Cambridge Street, Manchester, M15 6FH, UK. E-mail luisa.fernandez@manchester.ac.uk

\section{Question: What is the effectiveness of local interventions used for the prevention and treatment of dry socket?}

Data sources The Cochrane Oral Health Group Trials Register, Cochrane Central Register of Controlled Trials (CENTRAL), Medline and Embase databases were searched together with reference lists of identified articles. Topic experts and organisations were also contacted. Study selection Only randomised controlled trials were considered and there were no restrictions regarding language or date of publication. Data extraction and synthesis Data abstraction and risk of bias assessment were conducted in duplicate and Cochrane statistical guidelines were followed. The GRADE tool was used to assess the quality of the body of evidence.

Results Twenty-one trials with 2570 participants were included. Eighteen trials (2376 participants) related to prevention and three to treatment (194 participants). Six studies were at high risk of bias, 14 of unclear risk and one study at low risk.

There was moderate evidence (four trials, 750 participants) that chlorhexidine mouthrinses $(0.12 \%$ and $0.2 \%$ concentrations) both before and after extraction(s) prevented approximately $42 \%$ of dry socket(s) with a RR of 0.58 (95\% Cl 0.43 to $0.78 ; \mathrm{P}<0.001)$. The number of patients needed to be treated $(0.12 \%$ and $0.2 \%)$ with chlorhexidine rinse to prevent one patient having dry socket (NNT) was 232 (95\% Cl 176 to 417$), 47$ ( $95 \% \mathrm{Cl} 35$ to 84 ) and 8 (95\% Cl 6 to 14 ) at prevalences of dry socket of $1 \%, 5 \%$ and $30 \%$ respectively.

Thee was moderate evidence (two trials, in 133 participants) that placing chlorhexidine gel (0.2\%) after extractions prevented approximately $58 \%$ of dry socket(s) with a RR of 0.42 (95\% Cl 0.21 to $0.87 ; \mathrm{P}=0.02)$ with NNT of 173 ( $95 \% \mathrm{Cl} 127$ to 770$), 35$ (95\% Cl 25 to 154$)$ and $6(95 \% \mathrm{Cl} 5$ to 26$)$ at prevalences of dry socket of $1 \%, 5 \%$ and $30 \%$ respectively. There was insufficient evidence to determine the effects of other intrasocket preventive interventions or interventions to treat dry socket.

Conclusions There is some evidence that rinsing with chlorhexidine $(0.12 \%$ and $0.2 \%)$ or placing chlorhexidine gel $(0.2 \%)$ in the sockets

This paper is based on a Cochrane Review published in the Cochrane Library 2011, issue 12 (see www.thecochranelibrary.com for information). Cochrane Reviews are regularly updated as new evidence emerges and in response to feedback, and the Cochrane Library should be consulted for the most recent version of the review. of extracted teeth, provides a benefit in preventing dry socket. There was insufficient evidence to determine the effects of the other 10 preventative interventions each evaluated in single studies. There was insufficient evidence to determine the effects of any of the interventions to treat dry socket. The present review found some evidence for the association of minor adverse reactions with use of $0.12 \%, 0.2 \%$ and $2 \%$ chlorhexidine mouthrinses, though most studies were not designed to detect the presence of hypersensitivity reactions to mouthwash as part of the study protocol. No adverse events were reported in relation to the use of $0.2 \%$ chlorhexidine gel placed directly into a socket (though previous allergy to chlorhexidine was an exclusion criterion in these trials). In view of recent reports in the UK of two cases of serious adverse events associated with irrigation of dry socket with chlorhexidine mouthrinse, it is recommended that all members of the dental team prescribing chlorhexidine products are aware of the potential for both minor and serious adverse side effects.

Review It is beyond the scope of this review to describe and detail The Cochrane Collaboration. The reader can seek out more information at http://www.cochrane.org/. In brief, a systematic review supported and published by the Cochrane group represents the gold standard to support clinical decision-making.

\section{Commentary}

In this particular review, the authors have undertaken the analysis of a very difficult topic, the prevention and treatment of alveolar osteitis (AO) using local measures. 'While most clinicians know AO when they see one, for purposes of analysis, the working definition can be quite variable, making comparisons across studies difficult. The reported frequencies of $\mathrm{AO}$ range from $0-37.5 \%$. The wide range of estimates may be due to differences in the studies' working definition of $\mathrm{AO}$. In this report, diagnostic criteria for AO were not listed. Each study included had the diagnostic criteria agreed upon prospectively. It is not clear, however, that the definitions of AO were the same across studies, and combining data may not be appropriate. Given the unknown frequency of AO, a strength of this review was estimating the treatment effect across several possible frequencies of $\mathrm{AO}$, ie $1 \%, 5 \%$, and $30 \%$.

The results of this study suggest that the use of chlorhexidine mouthrise or intrasocket application have a minimal to marked effect on decreasing the frequency of $\mathrm{AO}$, ie the number needed to treat ranged from 232 (mouthrinse at a 1\% prevalence of $\mathrm{AO}$ ) to six (intrasocket application at a $30 \%$ prevalence of AO). Despite the authors' cautions to the contrary, which seems overestimated 
in this reviewer's opinion, the risk of adverse effects from brief exposures to chlorhexadine is minimal.

The authors reviewed the use of chlorhexidine in the prevention of $\mathrm{AO}$, but failed to offer any opinions regarding other local treatments to prevent or treat $\mathrm{AO}$ other than to say that the data were "insufficient" to determine their effects. Failing to offer any recommendations based on these other single study treatments that were all randomised clinical trials is an abrogation of the reviewers' responsibilities. Evidence-based clinical decision-making is not making decisions with the 'best' evidence. It is making clinical decisions with the 'best' evidence that is available. As such, the reviewers leave it to the reader to make some sense of the other 10 intrasocket interventions to prevent $\mathrm{AO}$ and the five interventions for treating $\mathrm{AO}$. Fortunately, the reviewers outlined these other studies with enough detail to make some tentative observations. Based on the material provided (including additional tables), many studies were plagued by inadequate power to detect a difference between local treatments to prevent $\mathrm{AO}$. In two out of three studies, intrasocket application of antibiotics (metronidazole or clindamycin) were statistically effective in reducing $\mathrm{AO}$ frequency and the direction of the treatment effect was all in favour of the antibiotic groups, suggesting a promising area of future research efforts.

Treating AO continues to be the bane of both patients and clinicians. The reviewers cited five studies using topical anesthetic, Alvogyl (a propriatery AO paste), Salicept (a proprietary mouthrinse or oral patch used for the relief of pain and management of inflammatory oral lesions or injuries), Alvogyl versus Salicept, or metronidazole. This report failed to offer any additional new information to improve patient outcomes for those with AO.

Based on the results of this report, this reviewer has changed perioperative treatment to include pre- and post-operative rinses of $0.12 \%$ chlorhexidine.

\section{Tom Dodson}

Department of Oral and Maxillofacial Surgery, Center for Applied Clinical Investigation, Massachusetts General Hospital,

Boston, USA

Evidence-Based Dentistry (2013) 14, 13-14. doi:10.1038/sj.ebd.6400913 